\title{
Weight Loss Maintenance for 2 Years after a 6-Month Randomised Controlled Trial Comparing Education-Only and Group-Based Support in Japanese Adults
}

\author{
Yoshio Nakata ${ }^{a, b}$ Masafumi Okada ${ }^{a, b}$ Koichi Hashimoto ${ }^{a, b}$ \\ Yoshinori Harada $^{a}$ b Hirohito Sone ${ }^{c}$ Kiyoji Tanaka ${ }^{d}$ \\ ${ }^{a}$ Faculty of Medicine, University of Tsukuba, Tsukuba, Japan; ${ }^{b}$ Tsukuba Critical Path Research \\ and Education Integrated Leading Center (CREIL), University of Tsukuba, Tsukuba, Japan; \\ 'Department of Internal Medicine, Niigata University Faculty of Medicine, Chuoh-ku, Japan; \\ ${ }^{d}$ Faculty of Health and Sport Sciences, University of Tsukuba, Tsukuba, Japan
}

\section{Key Words}

Behavioural interventions - Dietary treatment - Metabolic syndrome - Obesity management . Physical activity

\begin{abstract}
Objective: Our previous study, a 6-month randomised controlled trial, demonstrated that a group-based support promoted weight loss as compared to an education-only intervention. The purpose of this study was to examine weight loss maintenance for 2 years. Methods: Originally, 188 overweight Japanese adults, aged 40-65 years, were randomly assigned to 3 groups: control, education-only or group-based support. After the 6-month intervention, 125 participants in the education-only and the group-based support groups were followed up for 2 years. The primary outcome was the amount of weight lost. The participants were retrospectively grouped into quartiles of percent weight loss for secondary analyses. Results: At the end of follow-up, the amount of weight lost in the education-only and the group-based support groups was the same $(3.3 \mathrm{~kg})$. Secondary analyses using data of those who completed the study $(n=100)$ revealed that the participants in the highest quartile of percent weight loss significantly increased their step counts and moderate-to-vigorous physical activity compared with the lowest quartile. No significant differences were observed in the energy intake among the four groups. Conclusion: The effects of group-based support disappear within 2 years. Increasing physical activity may be a crucial factor for successful maintenance of weight loss.

(c) 2014 S. Karger GmbH, Freiburg
\end{abstract}


Nakata et al.: Weight Loss Maintenance for 2 Years after a 6-Month Randomised Controlled Trial Comparing Education-Only and Group-Based Support in Japanese Adults

\section{Introduction}

Excess body weight is a major public health concern. Globally, the mean BMI has increased since 1980 [1]. Between 1980 and 2008, individuals in Japan had a lower BMI than individuals in other high-income countries; the mean BMI of men increased from 22.1 to $23.5 \mathrm{~kg} / \mathrm{m}^{2}$ and that of women from 21.3 to $23.3 \mathrm{~kg} / \mathrm{m}^{2}$ [1]. Obesity is associated with high mortality [2] and high incidence of cancer [3], cardiovascular disease [4], coronary heart disease [5, 6] and diabetes [6]. Since April 2008, a nationwide health screening and intervention programme specifically targeting metabolic syndrome [7] is routinely conducted in Japan; however, the obesity statistics have not improved [8].

Treatment of obesity and maintenance of weight loss are challenging. Previous studies have examined the short- and long-term effects of various behavioural weight loss interventions. Although both diet [9] and exercise interventions [10] are important for more effective weight loss, diet intervention is prioritized over exercise intervention because of its larger effect on short-term weight loss [11]. A meta-analysis of changes in BMI after weight loss phases suggested that approximately $0.02-0.03 \mathrm{~kg} / \mathrm{m}^{2}$ of the weight lost per month is regained [9]. To attain successful weight loss maintenance, a few weight maintenance programmes have been developed $[12,13]$.

In a previous 6-month randomised controlled trial (RCT), we focused on providing educational materials (e.g., textbooks, notebooks and a pedometer) and implementing group-based support as effective individual components of a weight loss programme [14]. The weight lost in 6 months in the education-only group was $4.7 \mathrm{~kg}$ (95\% confidence interval (CI) 3.7-5.7 kg) and was significantly larger than that in the control group (2.9 kg; 95\% CI 1.9-3.9 kg), which was provided only a single motivational lecture. Weight loss in the group-based support group was $7.7 \mathrm{~kg}$ (95\% CI 6.7-8.8 kg) and was significantly larger than that in the control and the education-only groups. The RCT provided evidence that both educational materials and group-based support were effective components of a weight loss programme. However, no weight loss maintenance was observed after the intervention period.

Thus, in the present study, we conducted a 2-year, non-interventional, observational follow-up and annual follow-up measurements for participants in the education-only and the group-based support groups. Due to ethical concerns, we provided group-based support to the control group after the 6-month intervention period and did not include them in the follow-up measurements. A certain amount of weight regain might be inevitable because of the absence of weight maintenance programmes. However, a recent study demonstrated the effectiveness of motivational interviewing intervention at 12 months post intervention [15]. If the superiority of group-based support is conserved during the maintenance phase, it could be an essential approach on both short-term and long-term bases. However, if such superiority is not observed, a weight maintenance programme might be essential to maintain the effect of group-based support; thus, the factors responsible for successful weight loss maintenance could be explored. Therefore, in this study, we conducted a 2-year follow-up study, after completion of the 6-month intervention period, to examine and compare the effects of group-based support and education-only on weight loss maintenance.

\section{Material and Methods}

\section{Study Design and Participants}

The randomised trial method has been previously described $[14,16]$, and the protocol was registered with the University Hospital Medical Information Network Clinical Trials Registry (UMIN000001259). In brief, we conducted a 30-month RCT comprising a 6-month intervention between April and October 2009 and a 2-year, follow-up non-intervention that continued until October 2011; the study was conducted at the 
Nakata et al.: Weight Loss Maintenance for 2 Years after a 6-Month Randomised Controlled Trial Comparing Education-Only and Group-Based Support in Japanese Adults

Mito Kyodo General Hospital in Ibaraki, Japan. During the intervention phase, the participants were randomly assigned to one of the following 3 groups: control, education-only or group-based support. Considering an intervention-related group difference of $2.5 \pm 4.0 \mathrm{~kg}$ in the amount of weight lost at the end of 6 months, a two-sided alpha value of 0.0167 (with a Bonferroni's adjustment for post hoc tests), a power of $80 \%$ and an average attrition rate of $10 \%$, the required sample size was estimated to be more than 60 participants in each group (180 participants in total). Accordingly, 188 adults (145 women and 43 men) participated in the present study.

After the intervention phase had ended, the participants in the education-only and the group-based support groups underwent annual follow-up measurements during a 2-year follow-up period. Owing to ethical concerns, we provided group-based support to participants in the control group after the 6-month intervention period and did not include them in the follow-up measurements. Finally, 125 overweight adults (92 women and $33 \mathrm{men}$ ) from the education-only and the group-based support groups (62 and 63 adults, respectively) underwent follow-up. Assuming a two-sided alpha value of 0.05 , the number of participants in this study yielded $93 \%$ power to detect a group difference of $2.5 \pm 4.0 \mathrm{~kg}$ in the amount of weight lost at the end of 2-year follow-up. The participants received no financial compensation or gifts. The protocol was approved by the ethical committee of Mito Kyodo General Hospital and by the Institutional Review Board of the University of Tsukuba.

Participants were recruited through newspaper advertisements. The eligibility criteria for the participants included an age of 40-65 years, a BMI of $25-40 \mathrm{~kg} / \mathrm{m}^{2}$ and the presence of at least one of the following components involved in the diagnosis of metabolic syndrome, according to the Japanese criteria [17]: i) waist circumference $\geq 85 \mathrm{~cm}$ in men or $\geq 90 \mathrm{~cm}$ in women, ii) systolic blood pressure $\geq 130 \mathrm{~mm} \mathrm{Hg}$, iii) diastolic blood pressure $\geq 85 \mathrm{~mm} \mathrm{Hg}$, iv) triglyceride level $\geq 150 \mathrm{mg} / \mathrm{dl}(1.70 \mathrm{mmol} / \mathrm{l})$, v) high-density lipoprotein cholesterol level $<40 \mathrm{mg} / \mathrm{dl}(1.04 \mathrm{mmol} / \mathrm{l})$ and vi) fasting plasma glucose level $\geq 110 \mathrm{mg} / \mathrm{dl}(6.11 \mathrm{mmol} / \mathrm{l})$. The age and BMI ranges were based on the target for active support in the Japanese national intervention programme specifically targeted at metabolic syndrome [7]. The criteria for ineligibility included current or planned pregnancy, past history of coronary disease or stroke, and drug treatment for diabetes to avoid a potential influence on weight change [9]. To avoid a ripple effect on weight loss [18], participants whose cohabiting family member(s) participated in this study were also considered ineligible.

\section{Interventions}

After taking the baseline measurements in which the participants were assessed for eligibility, all participants attended a 2-hour, group-based, single motivational lecture conducted by an investigator (YN). The lecture consisted of the introduction of the Japanese national health screening and intervention programme conducted since April 2008 that specifically target at metabolic syndrome [7], combined cardiovascular risk factors and the outcome [19], and the target value for improving metabolic syndrome [20]. The participants also received typical weight control instructions on diet, exercise and behavioural changes. The recommendations included a calorie-restricted diet of 1,200 and 1,600 kcal/day for women and men, respectively [2022] and a minimum of $1,000 \mathrm{kcal} /$ week of increased physical activity [23]. All participants were encouraged to self-monitor their body weight every day.

After the motivational lecture, the participants were randomly assigned to one of the 3 groups using simple randomisation procedures involving computerised random numbers. The allocation data were generated by an investigator (MO) who had no contact with the participants or other staff members, and the data were maintained at a central secure location until completion of the motivational lecture.

At week 1 , the participants in the education-only and the group-based support groups attended a groupbased, 2-hour session in which they were provided with educational materials such as textbooks and notebooks containing information on daily diet and other lifestyle-related issues. The content of the textbooks and notebooks was based on the prior work of the investigators [20,24]. The dietary programme was based on the Four-Food-Group Point Method [14, 25]. Participants in the education-only and the group-based support groups were encouraged to modify their diet according to the information in the provided textbooks and were instructed to record their body weight, the content of meals and the daily step counts in the provided notebook. A pedometer (FB-720; Tanita, Tokyo, Japan) was also provided to motivate the participants to increase their physical activity [26].

The participants in the group-based support group attended a group-based, 2-hour session at weeks 2, $4,6,10,14,18$ and 22 . The frequency of support meeting was suggested to be one of the independent predictors of weight change [9]. In the present study, in the initial part of the intervention period, we reduced the frequency of the support meetings from every 2 weeks to every 4 weeks in order to provide the partici- 
Nakata et al.: Weight Loss Maintenance for 2 Years after a 6-Month Randomised

Controlled Trial Comparing Education-Only and Group-Based Support in Japanese Adults

pants information on the basics of changing behaviours. Each group-based support meeting was conducted by 3 staff members who were trained by the investigators (YN and KT). One staff member conducted lectures to explain the content of the textbooks, and 2 other staff members reviewed the participants' notebooks and advised them on their diet and other lifestyle factors at each session.

\section{Outcome Measures}

Data were collected at baseline and at months 3, 6, 18 and 30 in the hospital by trained hospital staff members who were blinded to the treatment assignment process.

The primary outcome measure was the amount of weight lost from baseline to 30 months. Wing and Hill [27] defined successful long-term weight loss maintenance as intentionally losing at least $10 \%$ of initial body weight and keeping it off for at least 1 year. According to the definition, the authors pre-determined the primary outcome as the 30 -month changes from baseline. Weight was measured to the nearest $0.05 \mathrm{~kg}$ using a calibrated digital scale (WB-150; Tanita). The participants wore light clothes and no shoes for the weight measurement. Height was measured to the nearest $0.1 \mathrm{~cm}$ on a wall-mounted stadiometer at baseline to estimate the BMI.

The secondary outcome measures were waist circumference, systolic and diastolic blood pressures, triglyceride level, high-density lipoprotein cholesterol level and fasting plasma glucose level. The waist circumference was measured to the nearest $0.1 \mathrm{~cm}$ at the umbilicus level using a flexible plastic tape, with the participants in the standing position. Blood pressure was measured using a manual sphygmomanometer, with the participants maintained in the seated position after a 20-min rest period. Two readings of systolic and diastolic blood pressures were recorded, and the average value was used for data analysis [28]. Approximately $10 \mathrm{ml}$ of blood was drawn from each participant (between 10:30 and 11:30 am) after a fasting period of more than $12 \mathrm{~h}$. Fresh samples were used for enzymatic analysis of triglycerides, and fasting plasma glucose level was assayed using glucose oxidase. The serum high-density lipoprotein cholesterol level was measured using heparin-manganese precipitation. Venous blood was analysed by an independent laboratory (Kotobiken Medical Laboratories, Ibaraki, Japan).

We measured the energy intake by using 3-day food records and physical activity using an Active style Pro three-axis accelerometer (HJA-350IT; Omron Healthcare, Kyoto, Japan). The participants were asked to record everything they consumed for 3 days, i.e., 2 weekdays and 1 weekend day. Foods were measured using standard measuring cups, spoons and digital scales. To ensure overall comparability, a skilled nutritionist who was blinded to the treatment assignment process analysed all of the food records. Furthermore, the participants were asked to wear the accelerometer on their waists throughout the day for 14 days. The accelerometer counted daily steps and estimated the intensity of physical activity, expressed as metabolic equivalents $[29,30]$. The devices were not worn when sleeping, while engaged in a water-based activity (e.g., bathing or swimming) or while participating in activities such as contact sports for safety reasons. Records obtained were defined as valid when a device was worn for at least $10 \mathrm{~h} /$ day [31]. If no acceleration signal was obtained over a 10-second time interval for 20 consecutive min or more, the period was defined as 'nonwear' [32]. When valid records were collected for more than 2 weekdays and 1 weekend day, we calculated the mean of the daily step counts and the total daily minutes of moderate-to-vigorous ( $\geq 3$ metabolic equivalents) physical activity (MVPA).

\section{Statistical Analysis}

The purpose of the initial part (6 months) of the present RCT was to examine the effectiveness of education-only and groups-based support using controls. Our previous study [14] used an intention-to-treat analysis, with the missing data replaced by the last observation carried forward. In the present follow-up study, we aimed at comparing the amount of weight lost from baseline to the end of follow-up in the educationonly and the group-based support groups. Thus, an intention-to-treat analysis, with the missing data replaced by baseline observation carried forward, was applied to the measures of body weight and related outcome variables. An unpaired $t$ test was used to examine the statistical significance of between-group differences, and a $\chi^{2}$ test was used to compare proportions. The participants were retrospectively grouped into quartiles of percent changes of initial body weight from baseline to the end of follow-up for secondary analyses, in order to explore the success factors for weight loss maintenance. Differences among the quartiles were examined using one-way analysis of variance for continuous variables and $\chi^{2}$ test for categorical variables. Data were analysed using IBM SPSS Statistics 20 (SPSS Inc., Chicago, IL, USA), with the level of statistical significance set at $5 \%$. 
Nakata et al.: Weight Loss Maintenance for 2 Years after a 6-Month Randomised Controlled Trial Comparing Education-Only and Group-Based Support in Japanese Adults

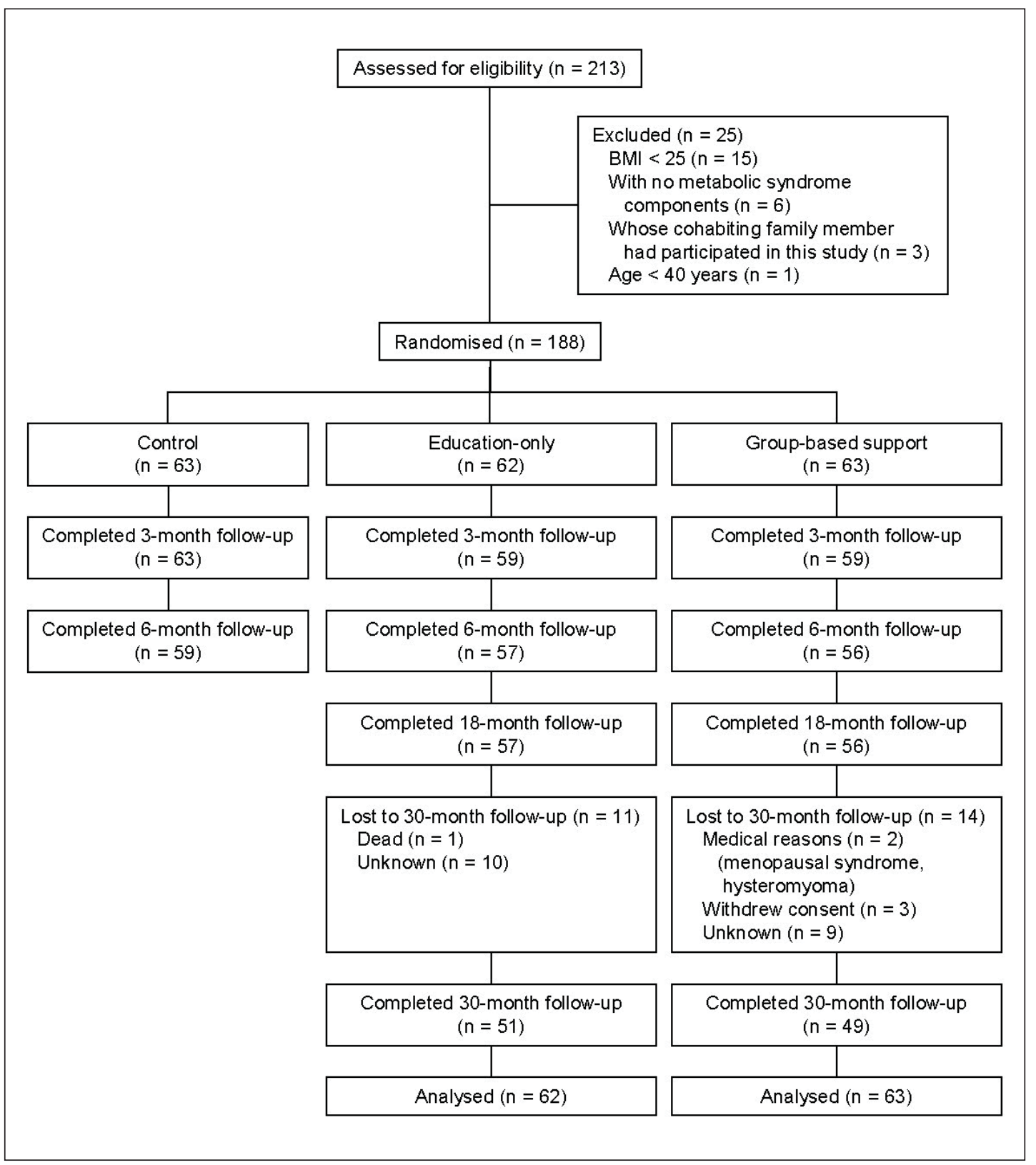

Fig. 1. Flow diagram of the participant progress through the 30 -month randomised trial.

\section{Results}

\section{Participants}

Figure 1 shows the number of participants through the course of the study. We recruited 222 participants between November 2008 and March 2009 and assessed 213 participants for eligibility ( 9 did not attend). After excluding 25 ineligible participants, 188 adults ( 145 women and 43 men) were enrolled in the intervention phase. After the intervention phase, participants in the education-only and the group-based support groups were followed up for 2 years. Table 1 provides the baseline characteristics of the followed-up participants. Signif- 
Nakata et al:: Weight Loss Maintenance for 2 Years after a 6-Month Randomised Controlled Trial Comparing Education-Only and Group-Based Support in Japanese Adults

Table 1. Baseline characteristics of followed-up participants by treatment assignment ${ }^{\mathrm{a}}$

\begin{tabular}{llll}
\hline & $\begin{array}{l}\text { Education-only } \\
(\mathrm{n}=62)\end{array}$ & $\begin{array}{l}\text { Group-based support } \\
(\mathrm{n}=63)\end{array}$ & p-value $^{\text {b }}$ \\
\hline Age, years & $51.7(6.8)$ & $50.7(6.7)$ & 0.414 \\
Women, no. (\%) & $41(66)$ & $51(81)$ & 0.059 \\
Current smoker, no. (\%) & $3(5)$ & $3(5)$ & 0.984 \\
Lipid-lowering therapy, no. (\%) & $10(16)$ & $3(5)$ & 0.033 \\
Antihypertensive therapy, no. (\%) & $16(26)$ & $15(24)$ & 0.796 \\
Height, cm & $160.0(9.0)$ & $159.1(7.2)$ & 0.577 \\
Weight, kg & $74.9(12.1)$ & $73.5(9.9)$ & 0.476 \\
BMI, kg/m & $29.2(3.8)$ & $29.0(3.0)$ & 0.670 \\
Waist circumference, cm & $100.7(7.9)$ & $99.2(7.3)$ & 0.269 \\
Systolic blood pressure, mm Hg & $131.2(14.5)$ & $131.9(16.4)$ & 0.793 \\
Diastolic blood pressure, mm Hg & $80.2(7.4)$ & $79.9(10.2)$ & 0.849 \\
Triglycerides, mmol/l & $1.57(0.71)$ & $1.26(0.59)$ & 0.009 \\
HDL cholesterol, mmol/l & $1.39(0.34)$ & $1.49(0.31)$ & 0.093 \\
Fasting plasma glucose, mmol/l & $5.50(0.96)$ & $5.25(0.59)$ & 0.079 \\
Energy intake, kcal/day & $2,181(417)$ & $2,169(414)$ & 0.880 \\
Step counts, step/day & $6,198(2,740)$ & $6,435(3,016)$ & 0.650 \\
MVPA, min/dayc & $86(30)$ & $93(35)$ & 0.191 \\
\hline
\end{tabular}

HDL = High-density lipoprotein; MVPA = moderate-to-vigorous physical activity .

a Data are presented as mean (standard deviation) unless otherwise specified.

${ }^{b} \mathrm{p}$ value of unpaired $t$-test or $\chi^{2}$ test at baseline between groups.

${ }^{c}$ Data from 123 eligible participants were available (61 and 62 in the education-only and the group-based support groups, respectively).

icant group differences were observed in the number of participants who were receiving lipid-lowering therapy and in the triglyceride levels. The attrition rates were $9.6 \%(12 / 125)$ and $20.0 \%(25 / 125)$ at months 18 and 30, respectively (fig. 1). The numbers of individuals lost to follow-up at 30 months were similar in both groups $(\mathrm{p}=0.531)$. The medical reasons for attrition during 30 months included menopausal syndrome and hysteromyoma that developed during the intervention phase. One participant died during the follow-up period, but the cause was not confirmed. No clinically significant adverse events related to participation in the trial occurred, as judged by the investigators.

\section{Intention-to-Treat Analyses}

The pattern of weight change is shown in figure 2. The mean weight loss during the 6-month intervention phase in the education-only and the group-based support groups was $4.7 \mathrm{~kg}(95 \% \mathrm{CI} 3.7-5.7 \mathrm{~kg})$ and $7.7 \mathrm{~kg}(95 \% \mathrm{CI} 6.7-8.8 \mathrm{~kg})$ respectively. The weight difference between the 2 groups reduced by half at 1 year ( $3.0 \mathrm{~kg}$ at 6 months to $1.5 \mathrm{~kg}$ at the 1 year) and disappeared at 2 years $(0.0 \mathrm{~kg} ; 95 \% \mathrm{CI}-1.7$ to $1.8 \mathrm{~kg})$. Table 2 shows changes in the secondary outcome measures, energy intake and physical activity. Most values (weight, BMI, waist circumference, systolic and diastolic blood pressures, high-density lipoprotein cholesterol level and energy intake) improved at 30 months as compared to the baseline values in each group, whereas no significant differences were observed between the 2 groups with respect to all variables. The stratified analysis by gender showed consistent results (data not shown).

\section{Secondary Analyses}

100 participants who completed the 30 -month assessment were retrospectively grouped into quartiles of percent weight loss from baseline to the end of follow-up for secondary 
Fig. 2. Pattern of change in body weight by treatment assignment. Each data point represents the mean value for all randomised participants, with missing data replaced by the last observation carried forward at months 3 and 6 and by the baseline observation carried forward at months 18 and 30. Error bars indicate standard errors. Points and error bars are jittered horizontally to improve visibility.

Nakata et al.: Weight Loss Maintenance for 2 Years after a 6-Month Randomised Controlled Trial Comparing Education-Only and Group-Based Support in Japanese Adults

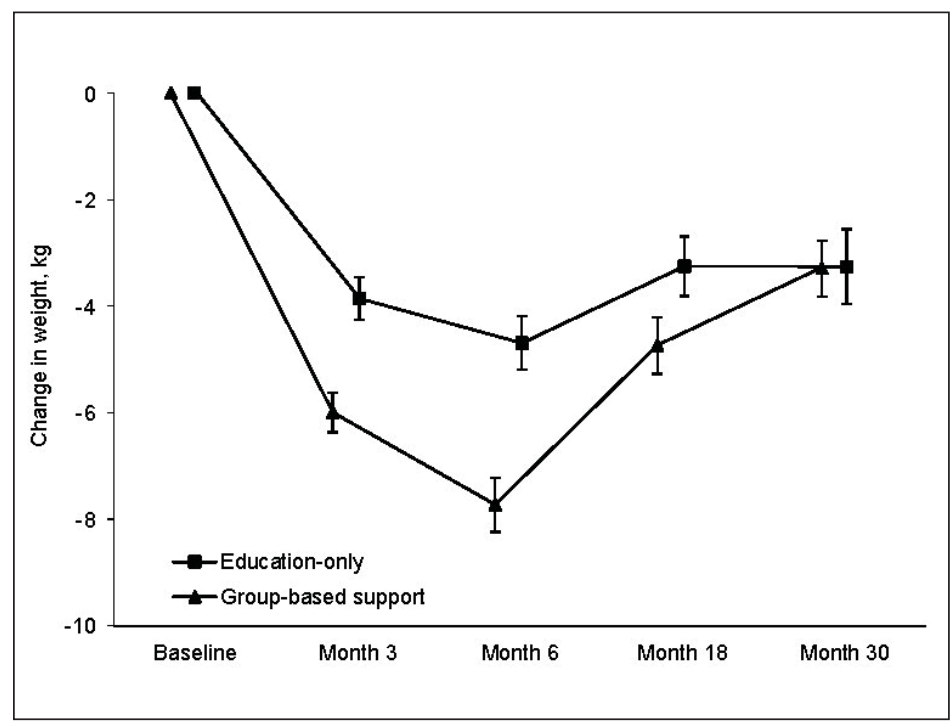

analyses (table 3). Baseline characteristics were not significantly different among the groups. Significant differences were observed in 30-month changes in waist circumference, systolic blood pressure, triglyceride level and high-density lipoprotein cholesterol level. The participants in the 4th quartile group who accomplished the most successful weight loss maintenance increased their step counts by 2,607 steps, which was higher than that observed in the other groups ( $p<0.05$ by Bonferroni's post hoc test). They also increased their MVPA by 21 $\mathrm{min} /$ day, which was higher than that in the 1st and 3rd quartile groups ( $\mathrm{p}<0.05$ by Bonferroni's post hoc test). In contrast to physical activity, 30-month changes in energy intake were not significantly different among the groups $(\mathrm{p}=0.602)$.

\section{Discussion}

The initial part of the present RCT (6-month intervention period) revealed that groupbased support is an effective method for promoting weight loss on a short-term basis. However, the present follow-up study revealed that the short-term effects disappeared after a 2-year non-intervention period.

A meta-analysis of changes in BMI after the weight loss phase suggests a regain of approximately $0.02-0.03 \mathrm{~kg} / \mathrm{m}^{2}$ per month during the maintenance phase [9]. The present study involved a 2-year (24-month) follow-up period. Accordingly, a regain of $0.48-0.72 \mathrm{~kg} / \mathrm{m}^{2}$ was estimated. In fact, the BMI of participants in the education-only group increased by $0.5 \mathrm{~kg} / \mathrm{m}^{2}$ during the maintenance phase, which approximately equalled the estimated value. On the other hand, the BMI of the group-based support group increased by $1.7 \mathrm{~kg} / \mathrm{m}^{2}$ during the maintenance phase. The regain in the group-based support group was approximately 3 times greater than that in the education-only group.

From the abovementioned data, it is evident that the group-based support was not effective after the 2-year follow-up period. This could be because group-based support appears to allow participants to interact with each other and provides short-term benefits in terms of achieving weight loss [33]. Further, the staff members motivated the participants. Although this is a strength of group-based support, it turns into a weakness if the programme is not continued. The intensive support might increase dependence on the staff members, and 
Nakata et al.: Weight Loss Maintenance for 2 Years after a 6-Month Randomised Controlled Trial Comparing Education-Only and Group-Based Support in Japanese Adults

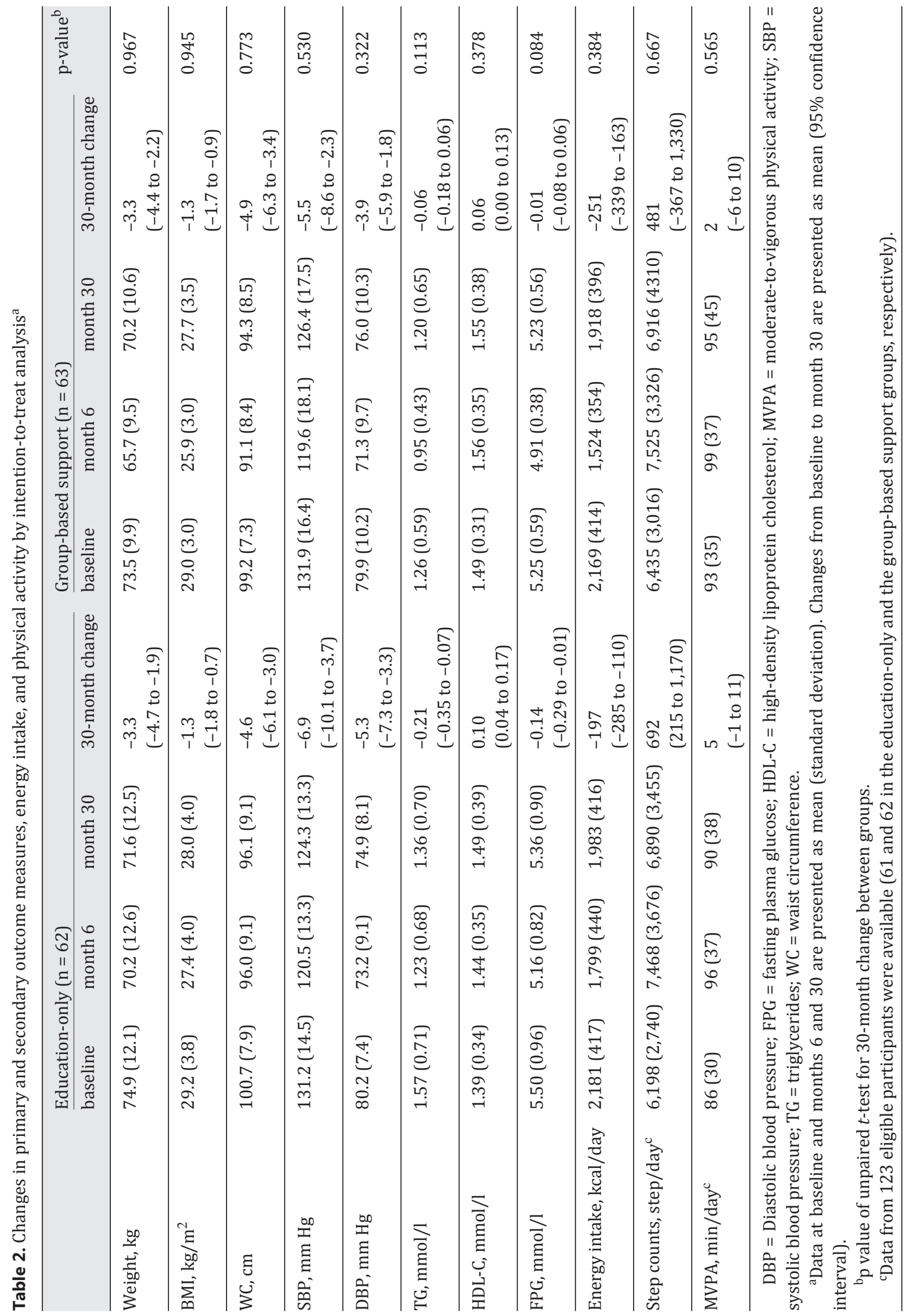


Nakata et al:: Weight Loss Maintenance for 2 Years after a 6-Month Randomised Controlled Trial Comparing Education-Only and Group-Based Support in Japanese Adults

Table 3. Baseline characteristics and changes in outcome measures based on quartiles of percent weight loss ${ }^{\mathrm{a}}$

\begin{tabular}{|c|c|c|c|c|c|}
\hline & \multicolumn{4}{|c|}{ Percent weight loss at 30 months } & \multirow[t]{2}{*}{$p$-value } \\
\hline & $\begin{array}{l}\text { 1st quartile } \\
(n=25)\end{array}$ & $\begin{array}{l}\text { 2nd quartile } \\
(n=25)\end{array}$ & $\begin{array}{l}\text { 3rd quartile } \\
(n=25)\end{array}$ & $\begin{array}{l}\text { 4th quartile } \\
(n=25)\end{array}$ & \\
\hline Age, years & $50.6(6.6)$ & $51.3(7.0)$ & $52.4(7.9)$ & $51.0(6.2)$ & 0.813 \\
\hline Women, no. (\%) & $16(64)$ & $21(84)$ & $20(80)$ & $16(64)$ & 0.230 \\
\hline Group-based support, no. (\%) & $10(40)$ & $14(56)$ & $12(48)$ & $13(52)$ & 0.704 \\
\hline Current smoker, no. (\%) & $1(4)$ & $1(4)$ & $2(8)$ & $0(0)$ & 0.414 \\
\hline Lipid-lowering therapy, no. (\%) & $2(8)$ & $3(12)$ & $4(16)$ & $2(8)$ & 0.778 \\
\hline Antihypertensive therapy, no. (\%) & $5(20)$ & $8(32)$ & $6(24)$ & $4(16)$ & 0.582 \\
\hline Height, $\mathrm{cm}$ & $162.1(9.7)$ & $157.5(5.8)$ & $157.5(6.6)$ & $161.4(8.2)$ & 0.074 \\
\hline \multicolumn{6}{|l|}{ Weight, kg } \\
\hline Baseline & $75.0(12.8)$ & $73.0(8.2)$ & $74.1(12.0)$ & $74.3(10.6)$ & 0.931 \\
\hline Changes & $+1.4(1.6)$ & $-1.8(0.7)$ & $-5.0(1.8)$ & $-10.9(4.2)$ & $<0.001$ \\
\hline \multicolumn{6}{|l|}{ BMI, $\mathrm{kg} / \mathrm{m}^{2}$} \\
\hline Baseline & 28.4 (2.9) & 29.4 (3.7) & 29.8 (3.9) & $28.4(2.3)$ & 0.312 \\
\hline Changes & $+0.5(0.6)$ & $-0.7(0.2)$ & $-2.0(0.6)$ & $-4.2(1.5)$ & $<0.001$ \\
\hline \multicolumn{6}{|l|}{$\mathrm{WC}, \mathrm{cm}$} \\
\hline Baseline & $99.4(7.0)$ & $100.2(8.8)$ & 101.8 (7.8) & 98.8 (5.3) & 0.500 \\
\hline Changes & $-0.4(4.4)$ & $-3.6(3.1)$ & $-7.4(3.3)$ & $-12.1(5.2)$ & $<0.001$ \\
\hline \multicolumn{6}{|l|}{ SBP, mm Hg } \\
\hline Baseline & $128.5(16.6)$ & $132.6(12.7)$ & $136.3(16.5)$ & $129.3(13.3)$ & 0.242 \\
\hline Changes & $-4.7(9.9)$ & $-3.2(14.9)$ & $-11.0(14.4)$ & $-12.0(13.0)$ & 0.045 \\
\hline \multicolumn{6}{|l|}{$\mathrm{DBP}, \mathrm{mm} \mathrm{Hg}$} \\
\hline Baseline & $78.2(8.2)$ & $80.9(7.6)$ & 79.8 (7.7) & $80.2(9.2)$ & 0.686 \\
\hline Changes & $-4.2(7.9)$ & $-5.7(9.6)$ & $-4.4(7.3)$ & $-8.7(8.9)$ & 0.222 \\
\hline \multicolumn{6}{|l|}{$\mathrm{TG}, \mathrm{mmol} / \mathrm{l}$} \\
\hline Baseline & $1.57(0.80)$ & $1.29(0.51)$ & $1.34(0.50)$ & $1.45(0.63)$ & 0.397 \\
\hline Changes & $-0.08(0.52)$ & $+0.00(0.51)$ & $-0.07(0.58)$ & $-0.52(0.58)$ & 0.004 \\
\hline \multicolumn{6}{|l|}{ HDL-C, mmol/l } \\
\hline Baseline & $1.41(0.32)$ & $1.49(0.28)$ & $1.48(0.28)$ & $1.41(0.40)$ & 0.738 \\
\hline Changes & $+0.02(0.21)$ & $+0.00(0.18)$ & $+0.04(0.24)$ & $+0.36(0.30)$ & $<0.001$ \\
\hline \multicolumn{6}{|l|}{$\mathrm{FPG}, \mathrm{mmol} / \mathrm{l}$} \\
\hline Baseline & $5.28(0.90)$ & $5.34(0.58)$ & 5.62 (1.13) & $5.31(0.65)$ & 0.460 \\
\hline Changes & $+0.03(0.30)$ & $-0.09(0.37)$ & $-0.07(0.56)$ & $-0.27(0.63)$ & 0.191 \\
\hline \multicolumn{6}{|l|}{ Energy intake, kcal/day } \\
\hline Baseline & $2,189(467)$ & $2,082(321)$ & $2,118(318)$ & $2,223(488)$ & 0.602 \\
\hline Changes & $-226(443)$ & $-219(274)$ & $-331(386)$ & $-345(352)$ & 0.492 \\
\hline \multicolumn{6}{|l|}{ Step counts, step/day ${ }^{\mathrm{c}}$} \\
\hline Baseline & $6,210(2,748)$ & $6,784(2,727)$ & $6,167(3,076)$ & $6,925(3,516)$ & 0.752 \\
\hline Changes & $-33(1,142)$ & $+379(2,924)$ & $+65(2,705)$ & $+2,607(4,000)$ & 0.005 \\
\hline \multicolumn{6}{|l|}{ MVPA, min/day ${ }^{\mathrm{C}}$} \\
\hline Baseline & $87(33)$ & $93(28)$ & $91(36)$ & $94(35)$ & 0.883 \\
\hline Changes & $-4(18)$ & $+5(28)$ & $-3(30)$ & $+21(41)$ & 0.018 \\
\hline
\end{tabular}

DBP = Diastolic blood pressure; FPG = fasting plasma glucose; HDL-C = high-density lipoprotein cholesterol; MVPA = moderate-to-vigorous physical activity; SBP = systolic blood pressure; TG = triglycerides; WC = waist circumference.

${ }^{\text {aD }}$ ata are presented as mean (standard deviation) unless otherwise specified.

${ }^{b} p$ value of one-way analysis of variance or $\chi^{2}$ test among groups.

'Data from 97 eligible participants were available $(25,25,23$ and 24 in the 1st, 2nd, 3rd, and 4th quartile groups, respectively). 
Nakata et al.: Weight Loss Maintenance for 2 Years after a 6-Month Randomised Controlled Trial Comparing Education-Only and Group-Based Support in Japanese Adults

the participants become prone to regaining weight. Therefore, the implementation of weight maintenance programmes after a group-based weight loss intervention might be essential to achieve successful weight loss maintenance.

The secondary analyses in the present study revealed that the participants who successfully maintained their weight loss over 30 months (highest quartile of percent weight loss) increased their step counts (2,607 steps/day) significantly more than those in the other groups (1st to 3rd quartiles). Furthermore, participants in the highest quartile group spent much more time in MVPA (21 min/day) than those in the 1st and 3rd quartile groups. Conversely, no significant difference was observed in the change in energy intake among the groups. These results suggest that increasing physical activity may be a crucial factor for successfully maintaining weight loss.

The American College of Sports Medicine Position Stand [34] recommended 250 $\mathrm{min} /$ week of physical activity for weight loss maintenance. However, the position stand also stated that no evidence from well-designed RCTs exists to judge the effectiveness of physical activity for prevention of weight regain after weight loss. A few previous studies facilitating maintenance of weight loss by focusing on increasing physical activity [35,36] concluded that the prescribed physical activity intervention did not influence the weight loss outcomes, but post hoc analysis showed that individuals sustaining successful weight loss performed more physical activity. Although improving adherence to the prescribed physical activity interventions is difficult, the effectiveness of increasing physical activity during the weight maintenance phase should be closely examined in future studies.

The major strength of this study is that it was a carefully designed long-term RCT with a relatively good retention rate. This study also had a few limitations. First, the study population included only Japanese participants, aged 40-65 years, who were willing and motivated, which limits the generalisation of the results. Additionally, most participants were women. Second, we could not follow up the control group of the initial part of the present RCT due to ethical concerns. Moreover, the attrition rate was $20.0 \%$ at 30 months. Although this figure is modest, an intention-to-treat analysis would have produced overly conservative results in hypothesis testing. Finally, secondary analyses suggest that increasing physical activity may be effective in successfully maintaining weight loss; however, RCTs are needed to demonstrate the effectiveness of the concept.

In conclusion, group-based support is an effective method for promoting weight loss on a short-term basis, butthe effect disappears in the absence of weightmaintenance programmes. For successful weight loss maintenance, additional approaches during the weight maintenance phase are necessary. Increasing physical activity may be crucial for successfully maintaining weight loss. Further RCTs are required to show that increasing physical activity can contribute to weight loss maintenance.

\section{Acknowledgments}

This study was supported by the Japan Agriculture Ibaraki Public Welfare Federation and by JSPS KAKENHI Grant Number 22700689. This study was also partly funded by the Meiji Yasuda Life Foundation of Health and Welfare. We wish to thank members of the Mito Kyodo General Hospital for their cooperation in data acquisition and members of the Tsukuba Critical Path Research and Education Integrated Leading Centre (CREIL) at the University of Tsukuba for their critical advice in conducting the study and data management during the study period. Special thanks to the participants of this study and Yuriko Sakairi for her cooperation in implementing the present study. 


\section{Disclosure Statement}

Nakata et al:: Weight Loss Maintenance for 2 Years after a 6-Month Randomised Controlled Trial Comparing Education-Only and Group-Based Support in Japanese Adults

The authors declare that there are no conflicts of interest.

\section{References}

1 Finucane MM, Stevens GA, Cowan MJ, Danaei G, Lin JK, Paciorek CJ, Singh GM, Gutierrez HR, Lu Y, Bahalim AN, Farzadfar F, Riley LM, Ezzati M; Global Burden of Metabolic Risk Factors of Chronic Diseases Collaborating Group (Body Mass Index): National, regional, and global trends in body-mass index since 1980:systematic analysis of health examination surveys and epidemiological studies with 960 country-years and 9.1 million participants. Lancet 2011;377:557-567.

-2 Flegal KM, Kit BK, Orpana H, Graubard BI: Association of all-cause mortality with overweight and obesity using standard body mass index categories: a systematic review and meta-analysis. JAMA 2013;309:71-82.

-3 Renehan AG, Tyson M, Egger M, Heller RF, Zwahlen M: Body-mass index and incidence of cancer: a systematic review and meta-analysis of prospective observational studies. Lancet 2008;371:569-578.

-4 Arnlöv J, Ingelsson E, Sundström J, Lind L: Impact of body mass index and the metabolic syndrome on the risk of cardiovascular disease and death in middle-aged men. Circulation 2010;121:230-236.

5 Bogers RP, Bemelmans WJ, Hoogenveen RT, Boshuizen HC, Woodward M, Knekt P, van Dam RM, Hu FB, Visscher TL, Menotti A, Thorpe RJ Jr, Jamrozik K, Calling S, Strand BH, Shipley MJ; for the BMI-CHD Collaboration Investigators: Association of overweight with increased risk of coronary heart disease partly independent of blood pressure and cholesterol levels: a meta-analysis of 21 cohort studies including more than 300000 persons. Arch Intern Med 2007;167:1720-1728.

-6 Tirosh A, Shai I, Afek A, Dubnov-Raz G, Ayalon N, Gordon B, Derazne E, Tzur D, Shamis A, Vinker S, Rudich A: Adolescent BMI trajectory and risk of diabetes versus coronary disease. N Engl J Med 2011;364:1315-1325.

7 Kohro T, Furui Y, Mitsutake N, Fujii R, Morita H, Oku S, Ohe K, Nagai R: The Japanese national health screening and intervention program aimed at preventing worsening of the metabolic syndrome. Int Heart J 2008;49: 193-203.

8 Ministry of Health, Labour and Welfare: Final Assessment of Healthy Japan 21 (in Japanese). www.mhlw.go.jp/ stf/houdou/2r9852000001r5gc-att/2r9852000001r5np.pdf.

-9 Dansinger ML, Tatsioni A, Wong JB, Chung M, Balk EM: Meta-analysis: the effect of dietary counseling for weight loss. Ann Intern Med 2007;147:41-50.

10 Curioni CC, Lourenço PM: Long-term weight loss after diet and exercise: a systematic review. Int J Obes (Lond) 2005;29:1168-1174.

11 Weinheimer EM, Sands LP, Campbell WW: A systematic review of the separate and combined effects of energy restriction and exercise on fat-free mass in middle-aged and older adults: implications for sarcopenic obesity. Nutr Rev 2010;68:375-388.

-12 Sherwood NE, Crain AL, Martinson BC, Anderson CP, Hayes MG, Anderson JD, Senso MM, Jeffery RW: Enhancing long-term weight loss maintenance: 2 year results from the Keep It Off randomized controlled trial. Prev Med 2013;56:171-177.

-13 Christensen JR, Overgaard K, Carneiro IG, Holtermann A, Søgaard K: Weight loss among female health care workers - a 1-year workplace based randomized controlled trial in the FINALE-health study. BMC Public Health 2012;12:625.

14 Nakata Y, Okada M, Hashimoto K, Harada Y, Sone H, Tanaka K: Comparison of education-only versus groupbased intervention in promoting weight loss: a randomised controlled trial. Obes Facts 2011;4:222-228.

-15 Hardcastle SJ, Taylor AH, Bailey MP, Harley RA, Hagger MS: Effectiveness of a motivational interviewing intervention on weight loss, physical activity and cardiovascular disease risk factors: a randomised controlled trial with a 12-month post-intervention follow-up. Int J Behav Nutr Phys Act 2013;10:40.

16 Nakata Y, Okada M: Effects of weight-loss tools and a group-based weight-loss support program: rationale and study design of a randomized controlled trial (in Japanese). Nippon Koshu Eisei Zasshi 2010;57:835-842.

17 The Examination Committee of Criteria for 'Metabolic Syndrome' in Japan: Criteria for 'metabolic syndrome' in Japan (in Japanese). Nippon Naika Gakkai Zasshi 2005;94:188-203.

18 Gorin AA, Wing RR, Fava JL, Jakicic JM, Jeffery R, West DS, Brelje K, Dilillo VG; Look AHEAD Home Environment Research Group: Weight loss treatment influences untreated spouses and the home environment: evidence of a ripple effect. Int J Obes (Lond) 2008;32:1678-1684.

19 Nakamura Y, Yamamoto T, Okamura T, Kadowaki T, Hayakawa T, Kita Y, Saitoh S, Okayama A, Ueshima H; NIPPON DATA 80 Research Group: Combined cardiovascular risk factors and outcome: NIPPON DATA80, 1980-1994. Circ J 2006;70:960-964.

20 Nakata Y, Okura T, Matsuo T, Tanaka K: Factors alleviating metabolic syndrome via diet-induced weight loss with or without exercise in overweight Japanese women. Prev Med 2009;48:351-356.

-21 Eto M, Ohkawara K, Sasai H, Tsujimoto T, So R, Matsuo T, Ohkubo H, Tanaka K: Efficiency of a free-living physical activity promotion program following diet modification for fat loss in Japanese obese men. J Nutr Sci Vitaminol (Tokyo) 2012;58:384-392. 
22 Tanaka K, Shigematsu R, Henwood T, Sasai H: Exercise, diet, and weight loss. J Phys Fitness Sports Med 2012; 1:457-465.

-23 Okura T, Nakata Y, Tanaka K: Effects of exercise intensity on physical fitness and risk factors for coronary heart disease. Obes Res 2003;11:1131-1139.

24 Tanaka K, Okura T, Shigematsu R, Nakata Y, Lee DJ, Wee SW, Yamabuki K: Target value of intraabdominal fat area for improving coronary heart disease risk factors. Obes Res 2004;12:695-703.

25 Kagawa A: The 'four-food-group-point-method' (in Japanese). J Kagawa Nutr Univ 1983;14:5-12.

26 Bravata DM, Smith-Spangler C, Sundaram V, Gienger AL, Lin N, Lewis R, Stave CD, Olkin I, Sirard JR: Using pedometers to increase physical activity and improve health: a systematic review. JAMA 2007;298:22963304.

27 Wing RR, Hill JO: Successful weight loss maintenance. Annu Rev Nutr 2001;21:323-341.

28 Chobanian AV, Bakris GL, Black HR, Cushman WC, Green LA, Izzo JL Jr, Jones DW, Materson BJ, Oparil S, et al; National High Blood Pressure Education Program Coordinating Committee: Seventh report of the Joint National Committee on Prevention, Detection, Evaluation, and Treatment of High Blood Pressure. Hypertension 2003;42:1206-1252.

29 Ohkawara K, Oshima Y, Hikihara Y, Ishikawa-Takata K, Tabata I, Tanaka S: Real-time estimation of daily physical activity intensity by a triaxial accelerometer and a gravity-removal classification algorithm. Br J Nutr 2011;105:1681-1691.

-30 Oshima Y, Kawaguchi K, Tanaka S, Ohkawara K, Hikihara Y, Ishikawa-Takata K, Tabata I: Classifying household and locomotive activities using a triaxial accelerometer. Gait Posture 2010;31:370-374.

-31 Troiano RP, Berrigan D, Dodd KW, Mâsse LC, Tilert T, McDowell M: Physical activity in the United States measured by accelerometer. Med Sci Sports Exerc 2008;40:181-188.

-32 Mâsse LC, Fuemmeler BF, Anderson CB, Matthews CE, Trost SG, Catellier DJ, Treuth M: Accelerometer data reduction: a comparison of four reduction algorithms on select outcome variables. Med Sci Sports Exerc 2005; 37:544S-554S.

33 Befort CA, Donnelly JE, Sullivan DK, Ellerbeck EF, Perri MG: Group versus individual phone-based obesity treatment for rural women. Eat Behav 2010;11:11-17.

34 Donnelly JE, Blair SN, Jakicic JM, Manore MM, Rankin JW, Smith BK; American College of Sports Medicine: American College of Sports Medicine Position Stand: Appropriate physical activity intervention strategies for weight loss and prevention of weight regain for adults. Med Sci Sports Exerc 2009;41:459-471.

-35 Jakicic JM, Marcus BH, Lang W, Janney C: Effect of exercise on 24-month weight loss maintenance in overweight women. Arch Intern Med 2008;168:1550-1559.

36 Jakicic JM, Otto AD, Lang W, Semler L, Winters C, Polzien K, Mohr KI: The effect of physical activity on 18-month weight change in overweight adults. Obesity (Silver Spring) 2011;19:100-109. 